2008

\title{
Dynamic Web Tools for Trigonometry
}

Steven J. Wilson

Johnson County Community College, swilson@jccc.edu

Follow this and additional works at: http://scholarspace.jccc.edu/mathtech

Part of the Numerical Analysis and Scientific Computing Commons, Other Mathematics Commons, and the Science and Mathematics Education Commons

\section{Recommended Citation}

Wilson, Steven J., "Dynamic Web Tools for Trigonometry" (2008). Innovations in Math Technology. 2. http://scholarspace.jccc.edu/mathtech/2

This Article is brought to you for free and open access by the Mathematics at ScholarSpace @ JCCC. It has been accepted for inclusion in Innovations in Math Technology by an authorized administrator of ScholarSpace@ JCCC. For more information, please contact bbaile14@jccc.edu. 


\title{
Dynamic web tools for trigonometry
}

\author{
Steven J. Wilson
}

Department of Mathematics, Johnson County Community College, 12345 College Blvd., Overland Park, KS 66210, USA

E-mail: swilson@jccc.edu

\begin{abstract}
In the last 20 years, computer technology having mathematical capability has been developed, improved, and become widely available, but textbook presentations are still largely free of any discussion that might require technology. Technology could be used in mathematical instruction for student drill and practice, for instructor demonstrations that promote conceptual understanding, or for the exploration of mathematical ideas, but software is often designed to be pedagogically generic, leaving its use to the creativity of the instructor. Technological solutions for local machines can be quite extensive, but cost and time constraints then limit availability for student use. The internet has the capability to provide mathematical instruction at all hours and in all places, with little financial investment by the student. This article describes the issues encountered and addressed by the author as he attempted to include self-authored web-based instructional units into a traditional trigonometry class.
\end{abstract}

Keywords: conceptual understanding; drill and practice; dynamic web tools; education; learning; mathematics technology; microsoft excel; teaching; trigonometry; variation of parameters; webMathematica.

Reference to this paper should be made as follows: Wilson, S.J. (2008) 'Dynamic web tools for trigonometry', Int. J. Continuing Engineering Education and Life-Long Learning, Vol. 18, Nos. 5/6, pp.698-707.

Biographical note: Steven J. Wilson is a Professor of Mathematics at the Johnson County Community College. He earned his BA in Mathematics and his MAT in Mathematics Education, both from the University of Chicago. He is the author of a Business Math textbook published by Kendall/Hunt. He was a co-recipient of the 2004 ICTCM Award for Excellence and Innovation with the Use of Technology in Collegiate Mathematics, and of a 2005 Innovation of the Year Award from the League for Innovation in the Community Colleges. Currently, he is the President of the Kansas Mathematical Association of Two-Year Colleges.

\section{Introduction}

The use of technology is promoted at the Johnson County Community College (JCCC). The JCCC Strategic Plan states that "innovation must be encouraged, with every result - positive or not - viewed as a learning experience" and that technology should be used to 'enhance learning' (JCCC, 2000). Being located in an affluent suburb of Kansas City and supported by local taxes, JCCC has the resources to foster innovation in the use 
of technology. The JCCC Math Resource Center has had computers available for student use since the late 1980s, all instructors have had desktop computers since the early 1990s, and most math classrooms were equipped with at least one computer station by the late 1990s. Both Microsoft Office (including the Excel spreadsheet program) and Wolfram Publishing's computer algebra system Mathematica have been available on the JCCC campus since the early 1990s. Although JCCC is by no means unique in this respect, many other of the US colleges have not had the same access to software that we have had.

The US textbook publishers have been at pains to respond to the ever-changing implications of technology use in mathematics education. At JCCC, we are currently using the fifth edition of a fairly common trigonometry textbook whose first edition appeared in 1984. The incorporation into the textbook of graphing calculator technology occurred in 2002 with the fourth edition, but then only with a brief note to be aware of screen settings, and radian vs. degree mode. The fifth edition is the first by this author to include a simulated graphing calculator screen. This textbook is considered quite traditional, and is well liked by the faculty at our college. It is easy to find other textbooks that incorporate graphing calculator use more fully, but rare is the textbook which discusses computer algebra systems at any length. Most of the US textbooks still focus on process and correct answers.

In 2001, Wolfram Publishing introduced webMathematica, an application which allows a computer user to interact with the computer algebra system Mathematica to obtain mathematical results through a form-driven web page. The software resides on a server; the user needs only a web browser. The terms of the Mathematica software license allowed JCCC to install and use webMathematica, and we immediately seized the opportunity. Alan Swarts, Director of Computing Support Services, led the technical installation. This author and Michael Martin, both Professors of Mathematics at JCCC, began writing web pages incorporating the product for use in their classrooms, and they soon referred to these creations as dynamic web tools. These efforts have been acknowledged by the educational community with multiple awards.

Due to a change in teaching assignment, the author began developing dynamic web tools for trigonometry in late 2004. The adjective dynamic suggests activity, and the use of this word is intended to refer to the activity of the user or learner, not simply motion in an animated image. Therefore, although the author has also created static web pages and animated images, the focus of this article is on the use of Excel spreadsheets and webMathematica pages in the teaching of trigonometry. Typically, each tool has, as its purpose, one of the following three goals: to promote drill and practice, to enable the exploration of the effect of variation in parameters, or to foster conceptual understanding. The entire collection of web pages and tools for trigonometry (both dynamic and static), including all of the tools discussed in this article, is available on the web at: http://staff.jccc.edu/swilson/trig/index.htm.

\section{Drill and practice}

Routine computations form the bulk of the exercises in the US textbooks, and much classroom time can be spent on them. Technology now allows an instructor to pass the monitoring of this repetitive task on to a machine. Various learning management systems are available which can deliver questions to a student, grade the answers and post the 
results to a gradebook, but the variety of question types may be limited. Using webMathematica, A set of drillmasters was created that perform the delivery and grading of questions in a way which promotes mastery of skills. When a student has completed an assigned drillmaster, the web page can be printed and submitted for credit. Students in online courses can capture the image and submit it by email for credit.

Each drillmaster will contain several common elements. Instructions to the student and credits appear on every page. Figure 1 shows fields for student input, and buttons for checking results and proceeding to the next problem from a typical drillmaster. Figure 2 from a typical drillmaster shows a table of results, the date and time of the last interaction with the web server, and a session ID. The session ID is based partially on the date and time the session began, and partially randomly generated, making it highly unlikely that two users would ever receive the same session ID.

Figure 1 Basic trig drill and practice

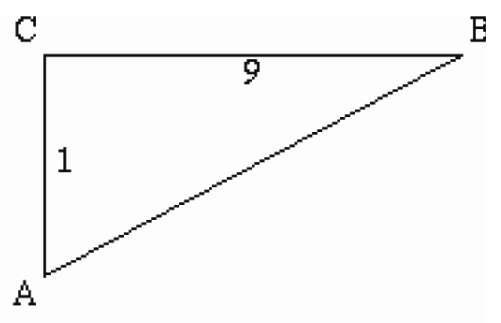

$$
\begin{aligned}
& \sin \mathrm{B}=1 / \mathrm{Sqr}[\mathrm{82}] \quad \text { Correct } \\
& \cos \mathrm{B}=9 / \mathrm{sqr}[\mathrm{82}] \quad \text { Correct } \\
& \tan B=0.111111 \quad \text { Wrong } \\
& \cot \mathrm{B}=9 \quad \text { Correct } \\
& \sec B=\square \quad \text { Wrong } \\
& \csc B=\sqrt{\operatorname{Sqt}(82) / 9} \quad \text { Wrong }
\end{aligned}
$$

\section{Check it New Problem}

Figure 2 Results from a typical drillmaster session

$\begin{array}{rll}\text { SESSION RESULTS } & \text { TOTAL } & \text { LAST } 10 \\ \text { Problems Displayed: } & 15 & 10 \\ \text { Right First Try: } & 10 & 6 \\ \text { Eventually Right: } & 12 & 8 \\ \text { Wrong: } & 2 & 1 \\ \text { Skipped: } & 1 & 1 \\ \text { Total ittempts: } & 16 & 11 \\ \text { Elapsed Time: } & 78 & 56\end{array}$

Date and Time: $2007 / 10 / 9,1259: 34.575553$

Session D: 9820636094.20071009125816 .6227 
The six trigonometric ratios can be defined in several different but essentially equivalent ways, the most basic definitions being through right triangles, through a point on the terminal side of an angle, and through a point on the unit circle. Each of these approaches is foundational, and the US students of trigonometry are expected to know all of them. To address these approaches, Three web tools were created, the Right Triangles Trig Ratios Drillmaster, the Standard Position Trig Ratios Drillmaster and the Special Angle Trig Values Drillmaster. The first of these is pictured in Figure 1.

In general, students have reacted favourably to the drillmasters. They receive immediate feedback, and can repeat the assignment until they have mastered the skill. Negative student reactions have occurred from three aspects of these assignments. Some students find it difficult to replace parentheses in functions with brackets, as required for correct Mathematica function notation (although code was included that would allow functions to be written in all lower case characters, thus partially simplifying the syntax of the software). Some students would rather use decimals, but the drillmaster requires fractions by our conscious design. And some students would prefer partial credit for answering some of the six functions correctly, but the drillmaster seeks mastery of all six, again by design. However, if the objective is mastery of the basic sine and cosine values, a fourth tool, the Special Angle Sine and Cosine Values Drillmaster, will request only one sine or cosine function at a time.

Another aspect of trigonometry that lends itself to the use of drillmasters is the conversion between alternate measurements of angles. This conversion was addressed with two drillmasters, the Converting Radians to Degrees Drillmaster, and the Converting Degrees to Radians Drillmaster. Each drillmaster gave the students a single special angle in either degrees or radians, and asked them to convert it to the other measure. These two drillmasters also received much favourable student reaction. Unlike the other drillmasters, neither required function notation nor multiple answers. When I assigned these to students, I required a high percentage of correct answers in a small amount of time. Some complained about the time restrictions, but as one student shared, 'Mr Wilson wants us to know these.'

To address the connections between the graph and the algebraic expression of a sine function, the Sine Graph Characteristics Drillmaster was created which gave the students a graph and an equation, and requested the amplitude, period and vertical translation. The results of this drillmaster were less satisfactory. The drillmaster purposely did not ask for the phase shift, since for each graph many different equations are possible, each with a different phase shift. The standard form of a polynomial expression in Mathematica is ascending order, rather than descending, which causes many students to stumble. Some may argue that this is actually a good thing, because students ought to pay careful attention to the details of a function, but most students react negatively. And although the process is formulaic and the approach of this drillmaster quite traditional, it is this author's opinion that some topics should be approached with an orientation toward conceptual understanding, not process.

The largest pedagogical benefit of the drillmasters seems to be a reduction in the class time required to practice basic skills. Standards developed by the American Mathematical Association of Two-Year Colleges state that "the use of technology, coupled with a decreased emphasis in some traditional content areas, should provide the time that is needed to implement the needed reforms in mathematics education" (AMATYC, 1995). 
Technically, the development of the drillmasters was both easy and tedious. The extensive language of Mathematica allowed code which automatically tests for equality of expressions, without the need for writing code to parse mathematical expressions. Most of the code for each drillmaster was to perform the bookkeeping related to displaying the results from each student session.

\section{Variation of parameters}

According to the Mathematical Association of America, students should 'see the graphical effects of varying parameters' and should study 'the effects of manipulating parameters on classes of functions' (MAA, 2004). For this purpose, the author created dynamic web tools using Microsoft Excel. For trigonometry, these included Curvilinear Coordinates and the Sine Function. In this tool, whose input is pictured in Figure 3, the student has the ability to choose a coordinate system and move sliders that affect the basic parameters of the sine function. Figure 4 shows an example of the output provided by this tool. The graph changes in real time, as the sliders are moved.

Figure 3 Typical controls of an Excel-based dynamic web tool

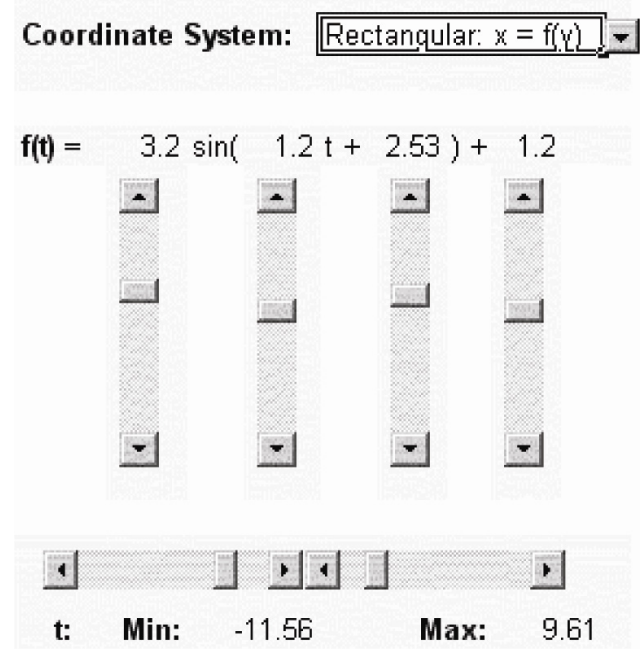

This web tool offers the ability to display a function or its inverse relation (which is not a function) in one of five coordinate systems: rectangular, polar, parabolic, elliptic and bipolar. Most students of trigonometry have no difficulty understanding the parameters of a sine function in a rectangular coordinate system. Interestingly, misunderstandings abound when viewing the inverse sine relation in the same system. For the inverse graph, students will often incorrectly identify the phase shift of a wave, having concentrated on the horizontal nature of its translation in the standard function setting. Polar graphs of the sine function are often taught in the course, but students are rarely exposed to the polar graph of a tangent or secant function. Opportunities for mathematical exploration abound for the gifted or curious student. The author has also created analogous tools for exploring the tangent function and the secant function. 
Figure 4 Changing the parameters of a polar sine function

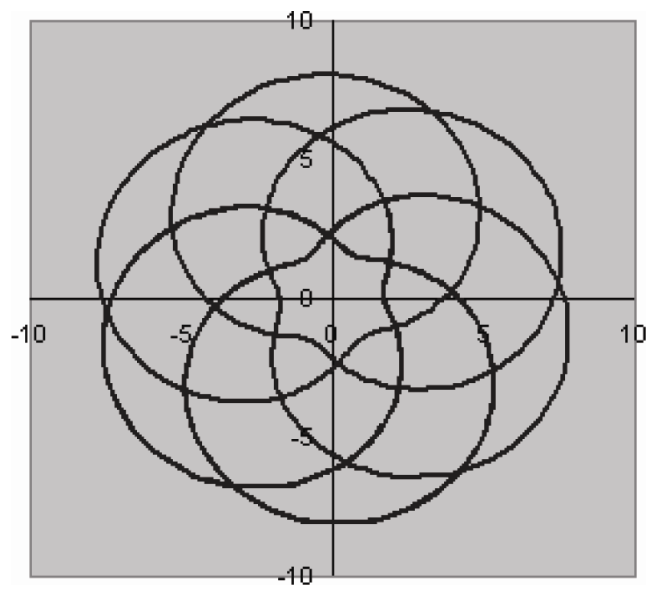

The Excel-based dynamic web tools exhibited many positive pedagogical attributes. The immediate response provided by the use of an ActiveX macro in Excel is very effective in making the connection between the parameter and the graphical characteristic. The similarity of all graphs generated by a particular tool helps teach the notion of a class of functions having the same attributes. The tool is useful both for instructor demonstration and for student exploration.

On the other hand, the use of Excel does have some disadvantages. Computers can be purchased in the USA which do not have the Microsoft Office suite pre-installed, so some students are unable to use the Excel-based dynamic web tools at home, and must use computers on our campus. Furthermore, the ActiveX controls are automatically disabled when the macro security control setting is high, which is the default setting when the software is shipped by Microsoft. Instructions must be provided to the student to change this setting.

The development of an Excel-based web tool is technically easy. Both the ActiveX controls and the graphs are provided by Microsoft, and each is easily linked to spreadsheet cells. The entire tool is easily saved as a web page.

Parameter variation is also possible in the webMathematica environment. The Rose Analyzer and the Limacon Analyzer are two such examples. In each case, the student provides the parameters to a polar equation and presses a button to display the graph. In a third tool, the Lissajous Curve Analyzer, the student provides the parameters of a set of parametric equations to see the graph.

Polar and parametric equations are introduced in most of the US trigonometry textbooks, but usually at a basic level. Roses are often introduced with positive integer coefficients of the variable, as they are the easiest to discuss in a non-technological environment, although at least one textbook (Larson, Hostetler and Edwards, 2005) does consider other coefficients in exploratory exercises. Another textbook (Lial, Hornsby and Schneider, 2005) introduces Lissajous curves as exercises in a discussion of parametric equations. Prior to computer tools, a student would have needed more than average curiosity to go beyond the usual parameter values, but technology now allows even the average student to obtain more interesting results. 
Having created the ability to study the rose curve with both Excel and webMathematica tools, some direct comparisons can be made. Whereas Excel used sliders to change the values of the parameters, webMathematica requires the student to fix a parameter value and press a button, making the connections between the parameter and the graphical characteristic more difficult to discern. On the other hand, when using the webMathematica tool the student must be sufficiently engaged in the mathematics to determine appropriate parameter values before obtaining a graph, whereas Excel will allow the student to explore the graphs without thinking mathematically until an explanation is required.

The development of the webMathematica-based dynamic web tools which explore parameter variation was comparatively easy. The Mathematica code is fairly basic, and is embedded in a simple HTML form. The technical difficulty lies in setting up the web server to process the JSP files.

\section{Conceptual understanding}

The Mathematical Association of America stated that students should 'possess conceptual understanding of the required mathematics' (MAA, 2004). Furthermore, the American Mathematical Association of Two-Year Colleges stated that

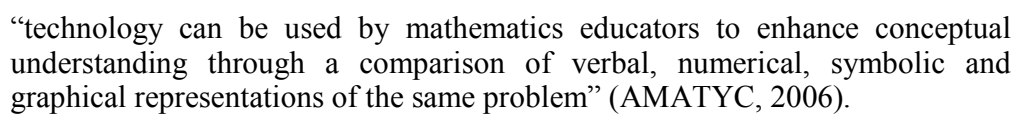

One dictionary definition of concept is 'an abstract or generic idea generalised from particular instances' (Webster's New Collegiate Dictionary, 1975). The variation of parameters of Section 3 fit these descriptions. Our investigations generated many particular instances that pointed toward the abstract idea or concept of a class of functions. Many other trigonometric concepts can be similarly motivated.

Many students are taught the relationship of the unit circle to values of the sine and cosine functions, but few of the US textbooks discuss the other four functions in terms of the unit circle. The Trig Values and the Unit Circle dynamic web tool provides this ability, and gives two different interpretations of each of the other four trig functions. The student provides an angle in either degrees or radians, presses a button, and receives several unit circle graphs of a style similar to Figure 5. These interpretations improve the student's ability to solve trigonometric equations, especially those involving the tangent function. The two solutions of a basic tangent equation on the interval $[0,2 \pi]$ are more easily recognised.

Fourier series is not typically a topic in trigonometry, but one textbook (McKeague and Turner, 2004) includes some exercises in which students explore the graph of a given partial sum of a Fourier series. This was implemented as the dynamic web tool Exploring Fourier Series. The student provides formulas for the coefficients, and a graph is produced. Although pregnant with possibilities, it is by no means obvious to this author how to produce the most interesting results, except through the traditional Fourier series formula. In a trigonometry class, this assignment was beyond the understanding of many students and did not achieve the desired results. 
Figure 5 Seeing the tangent and secant values of an angle

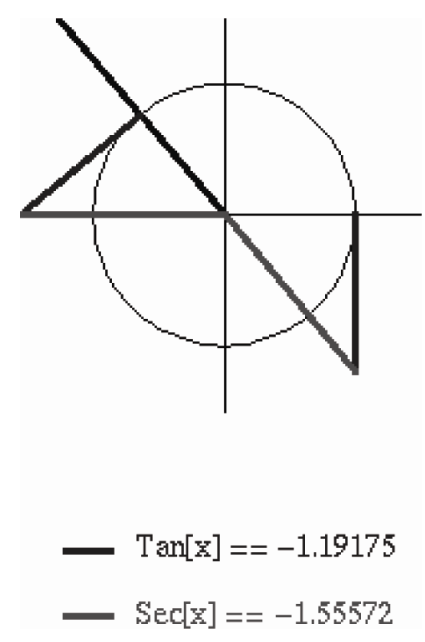

Rotations through the use of trigonometric functions in matrices can be easily demonstrated with technology. The 2D Matrix Transformations web tool allows students to enter values in a $2 \times 2$ matrix, and obtain several iterations of a figure transformed by that matrix. Figure 6 shows typical output from this tool. Similarly, the 2D Homogeneous Transformations web tool provides the student the opportunity to work with the $3 \times 3$ matrix in homogeneous coordinates to iteratively transform a $2 \mathrm{D}$ figure. Students are very interested in this concept because of its application to video game programming and animation, and are quite willing to spend a lot of time exploring its implications.

Figure 6 Rotations generated by a matrix transformation

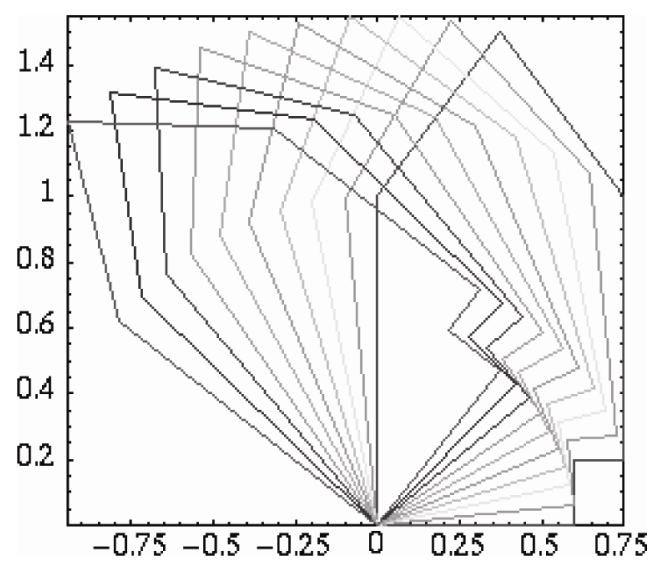

The connection between trigonometric and exponential functions can be studied with the Complex Exponential Function dynamic web tool. The student provides the parameters of an exponential function. Four graphs will be provided, as in Figure 7, so the student can see the mappings both from and to the real and imaginary axes. In this figure, the immediate consequences of Euler's Formula are instantly visible. The tool then provides 
two 3D graphs from the complex plane to the real and imaginary axes, followed by another pair of graphs showing the level curves of each 3D graph. Finally, Figure 8 shows the tool's final display of the intersections of the level curves of height zero, thus confirming the periodicity of the exponential function. With some effort, the best students in trigonometry can successfully understand the concept of a complex function, but this topic is difficult for the average student.

Figure 7 Four views of the complex exponential function
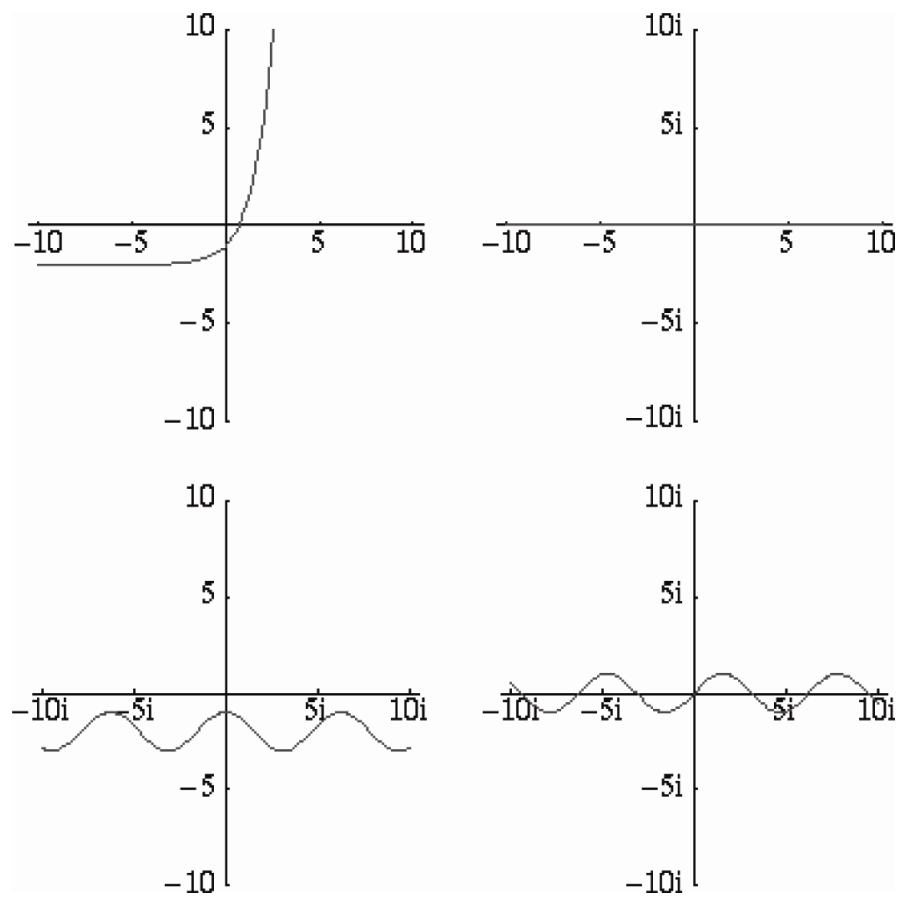

Figure 8 Seeing the periodicity of the complex exponential function

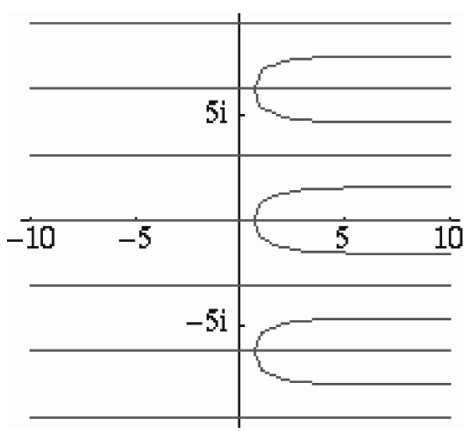




\section{Conclusions}

From a developer's point of view, Microsoft Excel and Wolfram's webMathematica have some very different characteristics. Excel may seem pervasive, but many students purchase computers at a discount and are unable to run Excel-based tools. The ActiveX controls used in these tools are unsigned, so Excel users may need to reset their macro security levels to use them. Students who have either issue may not be able to run the tools from their home computer, but they can be used in the JCCC computer labs. Since the webMathematica tools require only a browser to use, students can run the tools from virtually anywhere. Mathematica also has the ability to do almost anything mathematical, but the coding required can be extensive. The maintenance of the webMathematica server is not for the amateur, and JCCC relies heavily upon the services of the computer technical support staff.

Pedagogically, though still anecdotally, the author feels the incorporation of dynamic web tools into his trigonometry class has improved both student interest and the quality of student work. The webMathematica tools have provided excellent vehicles to monitor drill and practice, and to foster conceptual understanding in many situations. The realtime variation of parameters that Excel provides is very effective in establishing connections between equations and their graphs. A combination of the two products has yielded very positive results in the author's classroom.

\section{References}

AMATYC (1995) Crossroads in Mathematics: Standards for Introductory College Mathematics Before Calculus. Memphis, Tennessee: American Mathematical Association of Two-Year Colleges.

AMATYC (2006) Beyond Crossroads: Implementing Mathematics Standards in the First Two Years of College. Memphis, Tennessee: American Mathematical Association of Two-Year Colleges.

JCCC (2000) JCCC Strategic Plan. Overland Park, Kansas: Johnson County Community College.

Larson, R., Hostetler, R.P. and Edwards, B.H. (2005) Trigonometry: A Graphing Approach. Boston, MA: Houghton Mifflin.

Lial, M.L., Hornsby, J. and Schneider, D.I. (2005) Trigonometry (8th ed.). Boston, MA: Addison Wesley.

MAA (2004) Undergraduate Programs and Courses in the Mathematical Sciences: CUPM Curriculum Guide 2004. Washington, DC: Mathematical Association of America.

McKeague, C.P. and Turner, M.D. (2004) Trigonometry (5th ed.). Belmont, California: Brooks/Cole.

Webster's New Collegiate Dictionary (1975) Webster's New Collegiate Dictionary. Springfield, MA: G.\&C. Merriam Co. 